\title{
Fifth-generation research tools: Collaborative development with PowerLaboratory
}

\author{
DOUGLAS L. CHUTE and ROBERT F. WESTALL \\ Drexel University, Philadelphia, Pennsylvania
}

\begin{abstract}
The technology available for behavioral research limits the scope of the field. Yet we cannot expect personal computer manufacturers to be particularly concerned with real-time computing for such a niche market. The current, fourth generation of software tools is "old" technology, unable to take advantage of newer desktop technology like multimedia. Fifth-generation research tools, such as PowerLaboratory, will comprise software and hardware that overcome many of the limitations inherent in the fourth generation's tools. For example, PowerLaboratory's graphical programming interface resolves some of these limitations and also provides an advanced, verifiable, millisecond-accurate research tool that opens the discipline to multimedia as well as traditional stimuli. PowerLaboratory users have the option to create or link up a custom code extension (PLEX), and an Internet site provides for support and peer-reviewed protocol and PLEX publication. The purpose of this site is supporting open collaboration, which is seen as a requirement for advancing the technological capabilities of the discipline.
\end{abstract}

Studies of the history of technology generally show that a discipline is both simultaneously enriched and also constrained by its technological innovation. Psychology is no exception. In the 30 years that one of us (D.L.C.) has been engaged in psychological research, five generations of research tools can be broadly classified. Our purpose is not to present a technological history of psychology, even though such a need appears to exist, but rather to consider the problems that can be identified and the solutions that might be available as we progress from one generation to the next.

The first generation of psychology research tools began with the brass instruments of the end of the 19th century. Classic apparatuses like the Hippe Chronoscope and the Galton whistle were hand-made by artisans and crafts people to psychological specifications. For example, Max Meyer, while at the University of Missouri, had a quarter tone organ custom built for his psychophysiological studies in hearing. Like any technology, an earlier generation seldom completely disappears, and many such instruments had a broad enough demand that companies like Stoelting of Chicago continued production into the 1960s. Some instruments, such as mazes, Skinner boxes, polygraphs, and tachistoscopes, are still available from one of the few remaining psychological apparatus suppliers, Lafayette Instrument Company. In general, the benefit of this technology was precision in measurement. The constraints were extensive, owing to custom design, lack of flexibility or idiosyncratic design, and lack of fully auto-

Correspondence should be addressed to D. L. Chute, Neuropsychology, 47-110 Drexel University, Philadelphia, PA 19104 (e-mail: chute@ bc.drexel.edu).

Note: The authors have a direct financial interest in some of the software described in this paper-Editor mated control or procedural standardization. Until the commercial suppliers began to equip the larger departments after World War II, there was a significant technological impediment to replication and collaboration.

The second generation of psychology research tools had its first beginnings just prior to World War II with the advent of 28-V dc electromagnetic switching technology. This hardware was originally developed for the telephone system; psychologists learned to adapt it and "program" it with snap leads to create automated experimental and process control. Such direct hardware programming can be viewed as the beginning of computerization in the field, where relays and clock timers provided realistic accuracies to the 10th of a second. The somewhat more modern solid-state equivalents added an additional order of magnitude of accuracy. The primary benefit was automation, which permitted the presentation of a limited set of stimuli, the running of large numbers of subjects, and the recording and quantification of narrowly classified responses. The first widely available electromechanical calculators appeared toward the end of this second generation of technology and permitted the tedious, though now possible, use of more complex experimental designs and statistical analyses. One clear advantage of second-generation technologies was that researchers could design and build their own unique apparatus once they became proficient with snap lead circuit design. Such proficiency was no mean accomplishment, as many a former graduate assistant can testify. When such talent left a lab or graduated, there often was a long hiatus in productivity.

The constraints of second-generation technologies included the illusion of accuracy and the false confidence that it apparently provided. Contact point bounce and pitting, as in timers for tachistoscopes and clocks, typically limited their practical accuracy to only two decimal places. Most debilitating was the narrow scope of any existing 
facility and the difficulty researchers and students faced in designing and developing new protocols or quickly responding to innovations. Long learning and "debugging" curves with direct hardware wiring and programming were required. In Douglas Anger's lab, for example, a large room filled with scores of relay racks, thousands of modules, and tens of thousands of leads could program anything as long as it happened to the lights and the bars in a Skinner box. Although the modularity and customizability of relay controls empowered the individual laboratory as long as it had "focus," their complexity and idiosyncrasy tended not to empower individuals in those labs. As with later programming environments, service and maintenance began to appear as major limitations over time. Direct replication became a problem because independent laboratories shared little by way of a common technological base. Thus, at least to some extent, anticollaboration factors were caused by the technology and the unique way in which labs resolved technological problems. In part to redress these problems through better communication, Joseph Sidowski founded the journal Behavior Research Methods \& Instrumentation, the name of which was changed to Behavior Research Methods, Instruments, \& Computers in 1984.

The third generation of psychology research tools came from software applications on mainframes and minicomputers. Some laboratories, like that of Melvin Marx, could afford to enslave a minicomputer to managing experimental protocols and processes. However, the majority of uses for mainframes and minicomputers were statistical and analytical, although an early collection of papers in the field of computer applications under the series editorship of Herbert A. Simon and edited by Harold Borko (Borko, 1962) did include notions of neural modeling, simulation, and visualization. The advantages of these early computers in analysis over mechanical calculators outweighed their disadvantages in accessibility and in the arcane knowledge required for their use. As laboratory controllers, they primarily offered increased reliability over relays and programmability by paper punch tape or punch cards. Their cost in dollars and support personnel meant that few psychology departments, let alone laboratories, had unique control over them. Interestingly, about this time graduate training began to drop the foreign language requirement in exchange for some sort of computer or technology requirement. This came to mean a requirement to learn an interpreted programming language in most curricula.

Fourth-generation technology is contemporary. It can be broadly defined as the personalization of computers and their applications. In the early 1970s an Olivetti 101 programmable calculator could sit on a desktop and accept up to 64 lines of what would pass for BASIC on a magnetic card. Today, personal and specialized computers run statistical packages, control experiments, record biological data, and offer multimedia learning environments. They are affordable and available even to small departments and high schools. The advantages of flexibility, accessibility, and ubiquity seemed to make this technology an ideal mechanism to control experiments, collect data, model theories, and teach the discipline. It even became possible to offer a more or less complete behavioral laboratory on a disk or CD-ROM. The great problem was that the pace of movement of the underlying computer technology outstripped the ability of individual psychologists to supply and maintain suitable programs. Few faculty were both expert programmers and scientists. Early pioneers like Timothy Tyler, John Mueller, Russ Hurlburt, and others, no doubt, discovered that the service and maintenance of their efforts soon became overwhelming. Graduate curricula, in requiring formal programming courses, were not addressing the fundamental problem - the speed of change and the complexity of programming from "the ground up."

In the latter part of the 1980 s a concerted effort was made by ourselves, Tom Hewett, and other psychology software developers to promote a distribution and maintenance scheme for fourth-generation technology through quasi-commercial operations like Kinko's Academic CourseWare Exchange, Conduit, and Intellimation. These operations failed by the beginning of the 1990s, owing in part to poor economics and purchasing resistance in the discipline, but mostly to the "drying up" of faculty-developed software. Ultimately, most surviving psychology-specific software included the programs that had a viable commercial component so that they could afford to renew themselves. Of the two most widely distributed, MacLaboratory and Micro Experimental Laboratory, neither has reported being particularly profitable (Chute, 1993). Ironically, many constituencies in the discipline actively repudiate the commercialization of our core technological capabilities. In our opinion, this view, coupled with national research funding reductions, has been a limiting factor in software development in the field.

Although technologies were often considered the purview only of experimentalists, both the changes in the interests of the discipline and the accessibility of the technology have promoted the use of computers in all phases of psychology from the clinician, to the teacher, to the basic scientist. If we do not develop and maintain discipline-specific software tools, however, we will be dependent on repurposing software that is intrinsically unacceptable to psychological research. Manufacturers of personal computer hardware, operating systems, or presentation software have little interest in our need for real-time computing. For example, HyperCard for the Macintosh or ToolBook for Windows is inherently incapable of providing accurate timing even with programmed extensions. Psychology is such a minuscule software market that no general applications and, in fact, no operating systems can be expected to be designed with our unique needs for precision (Westall, Perkey, \& Chute, 1989). For example, in our fourth-generation application, Reaction Time, there are over 90,000 lines of code, much of which is designed to patch or work around hardware or operating systems that were never designed to conduct psychological research. This programming re- 
quirement has put most technically proficient software development beyond the reach of individuals who are not professional programmers.

\section{Fifth-Generation Research Tools}

Fifth-generation research tools will comprise software and hardware that overcome many of the problems of the previous generations of effort. These tools will have flexible, comprehensive graphical programming environments so that students and inexperienced users will be able to easily construct clinical, experimental, or teaching protocols. They will be platform independent. For the first time, experimental replication and collaboration should be readily possible worldwide. The only impediments will be "psychological," like the prevailing culture of independence and self-sufficiency, the resistance of parts of the discipline to technology, and so forth. For the first time, our technology will be transportable and shareable.

Fifth-generation research tools will also support open programmable extensions, miniapplications, or code modules that can be constructed by someone who has had a programming course or two. Thus the "little knowledge" that has been a dangerous thing in many homegrown laboratory applications will be avoided and graduate training in the field will be made more relevant. Code modules will plug into millisecond-accurate commercial "shells" designed for discipline-specific issues in psychology and related fields. Service and maintenance will be assured by professional programmers. Complete experiments and code module exchanges, training and support, and collaborative development will be facilitated electronically over the Internet. Throughout the history of psychology's use of technology, there has been a tradition of minimal collaboration in the development and use of our research tools. While journals have promoted communication, new collaborations will provide technology for the future of the discipline. Earlier attempts like Project BioQuest and PsyScope have had mixed success. Other disciplines - physics, for examplehave been more successful with collaboration, particularly in computer and supercomputer projects. We hope that centers like the Computers in Teaching Initiative (CTI) at the University of York (see Hammond, McKendree, \& Scott, 1996; Trapp, Hammond, \& Bray, 1996), as well as our own Brooks/Cole Technology Center at Drexel University, will augment the collaborative activities our discipline will require for its future success.

On the commercial side, companies like National Instruments are developing plug-in instrument solutions, while companies like Brooks/Cole Publishing are creating software solutions that clearly are evolving into fifthgeneration research tools. For our part, B/C PowerLaboratory has been developed with funding from Brooks/ Cole to further the research capabilities of the discipline by providing a graphical programming interface, verifiable, millisecond-accurate timing, support for various response options and experimental designs, and support for various stimuli, including multimedia. It provides full access for any number of plug-in code modules in the form of an extension called a PLEX (PowerLaboratory external), which can link to 13 different time points within PowerLaboratory's verifiably accurate millisecond timing shell. A version of PowerLaboratory will operate on the latest hardware running either Mac OS or Microsoft Windows.

To address the problem of successive generations of graduate students and researchers who cannot service or modify fourth-generation protocols (even those developed in their own labs), a visible graphical programming environment has been created for PowerLaboratory. Cut, copy, paste, and point, click, and shoot provide for the creation of a wide variety of research and clinical protocols. Features include easy editing access through various dialogs and palettes; tachistoscopic presentation (depending on CPU and monitor speed); remote synchronization and triggering; millisecond-accurate touch screen, mouse, or keyboard responses; multimedia stimuli; randomization; data filtering; feedback; and criterion branching. Users with unique research needs are given open access to B/C PowerLaboratory through a PLEXprogrammable code module. The B/C PowerLaboratory CD-ROM contains a manual along with sample experiment files, tutorials, various utilities, and a software development kit that includes APIs and sample code for building external code modules, if they are required.

\section{The PLEX}

Fifth-generation research tools will support code extensions that will consist of manageable units created and shared by researchers and laboratories. Already, productivity applications have their "plug-ins," Internet systems their "applets," spreadsheets their "macros," and scriptable authoring packages their "XCMDs." So that psychology technologies can grow, and so that the discipline is not dependent on too few developers, we expect fifth-generation psychology technology to also provide such access to accurate program "shells." To enable advanced researchers and programmers to achieve this higher degree of functionality and customization, the B/C PowerLaboratory run-time architecture provides an external programming interface. A PLEX is an executable code resource that can be attached to one or more pages of an experiment. For those familiar with Mac OS programming, a PLEX is similar in functionality to a system definition function that is typically used to control the behavior of, for example, windows (WDEF), menus (MDEF), and controls (CDEF). A PowerLaboratory external can access many of the internal global data structures of the run-time engine, including drawing layers, millisecond-resolute timers, and collected response data. The PLEX interface functions similarly in both the Mac OS and the Microsoft Windows environments.

A PowerLaboratory external function can be created in virtually any compiled (or assembled) language or development system that is capable of creating stand-alone code resources. PowerLaboratory will also support PLEX resources that contain processor-specific native code. 
Under Mac OS, for example, versions of B/C Power Laboratory are provided for both the $68 \mathrm{~K}$ and PowerPC architectures. By encapsulating code for both instruction sets within the PLEX, the programmer can achieve the maximum possible performance for either processor architecture.

\section{Availability}

Information, formal publication of experiment protocols, and PLEXs, as well as peer collaboration, are provided through the Internet at http://www.brookscole. drexel.edu/.

\section{Conclusion}

A new generation of psychological research technology is emerging that provides sophisticated experimental control for researchers as well as nonprogramming professionals. Flexibility - beyond that inherent in applications like PowerLaboratory-is facilitated through the creation of code modules with links to the millisecondaccurate engine. The distributability of run-time engines, their availability across platforms, their professional service and maintenance over time, and the collaborative support and exchange of experiments modules over the
Internet ensure that many of the problems of previous generations of technology will be resolved. Although this new technology provides new affordances to the discipline, the history of technological application assures us that it too will have its constraints.

\section{REFERENCES}

Borko, H. (1962). Computer applications in the behavioral sciences. Englewood Cliffs, NJ: Prentice-Hall.

CHUTE, D. L. (1993). MacLaboratory for psychology: Success, failures, economics, and outcomes over its decade of development. Behavior Research Methods, Instruments, \& Computers, 25, 180-188.

Chute, D. L., \& Westall, R. F. (1996). B/C PowerLaboratory. Pacific Grove, CA: Brooks/Cole.

Hammond, N., MCKendree, J., \& Scott, P. (1996). The PsyClE project: Developing a psychology computer-based learning environment. Behavior Research Methods, Instruments, \& Computers, 28, 336340.

TrapP, A., Hammond, N., \& Bray, D. (1995). Internet and the support of psychology education. Behavior Research Methods, Instruments, \& Computers, 28, 174-176.

Westall, R. F., Perkey, M. N., \& Chute, D. L. (1989). Millisecond timing on the Apple Macintosh: Updating Drexel's MilliTimer. Behavior Research Methods, Instruments, \& Computers, $21,540-547$.

(Manuscript received November 27, 1995; revision accepted for publication February 12, 1996.) 